\section{How to write a scientific paper in a way that may increase its chance of publication}

\author{
Michael Bachmann Nielsen, Lars Lönn \\ Department of Radiology, Rigshospitalet, Copenhagen, \\ Denmark
}

\section{Abstract}

In this paper we will look at some of the reasons for rejection and how you can use this to improve your chances for publication. There is no single best way to prepare and write a paper and there are many circumstances around each and every research work. But there is a clear need to follow some steps in the writing process which will make the struggle with the manuscript so much more enjoyable.

Structure your manuscript properly; however this is not always enough. In this paper we will give tips and tricks to how you should look at the different sections of your manuscript.

Decide early which journal you intend to submit to. We strongly suggest that the decision is taken even before the writing procedure is started. This facilitates the process of following guidelines for authors for the journal to which you are submitting.

The difficult part to accept - is your paper justified? Lack of clinical relevance will almost certainly lead to rejection. Also if it adds no new knowledge to the subject you should prepare yourself for rejection. It is often hard for the authors to realize this. Also remember the importance of writing your manuscript in fluent English.

\section{Introduction}

$\nabla$

If you have ever submitted a manuscript for possible publication you will most likely have experienced getting your paper rejected. Presumably you were annoyed about the decision and hold the reviewers and the editor responsible for not appreciating the content of your manuscript. However, the frustration of being rejected means you are forced to revise and resubmit, and there are surveys that even state that the process might boost your citation record [1].
Journals receive more manuscripts than they can publish and therefore needs to adopt a careful plan for what they consider best for the journal. Rejection rates are not available for all journals but occasionally referenced. High ranked journals in imaging such as Radiology report a rejection rate of approximately $85 \%$ of submitted manuscripts and often these are well written and of potential interest for the journal readers [2]. The low acceptance rate is in line with other high ranked journals such as Circulation, i.e. range $10-12 \%$ [3]. Many manuscripts are of excellent scientific quality, but may be rejected due to their needlelike focus and therefore not in scope for the universal mass or particular group of readers.

In this paper we will look at some of the reasons for rejection and what you can do to improve your chances for publication. There is no single best way to prepare and write a paper and there are many circumstances around each and every research work. But there is a clear need to follow some steps in the writing process which will make the struggle with the manuscript so much more enjoyable [4-5].

\section{The basics - structuring your ma- nuscript properly \\ $\nabla$}

Many authors wait until their manuscript is almost finished before the decision on journal is taken. But, even if you are an experienced author, the sooner you target your journal, the better. We strongly suggest that the decision is taken even before the writing procedure is started. This facilitates the process of following guidelines for authors for the journal to which you are submitting. These instructions for authors must be followed with military discipline, this cannot be stressed enough.
The outline is the most important step for your manuscript. The silhouette is the fundamental ground for the full paper. By using the step by step format, you will directly notice logistic gaps that need to be further addressed. Parallel to this work you need to express a central message of your paper. When you have decided upon the focused message in one or maximum two sentences, all your further texting efforts are done to support this overall statement(s).

The introduction should be kept short, in our opinion no more than three paragraphs and less than 400 words. Before the introduction is written, memorize your outline to see that the steps are logically ordered, having clarity and intelligibility, in short, that your plan is reasonable. First paragraph states the main message and issue, second concerns why your paper is needed (purpose and objective) and the third aims and hypotheses. To develop the introduction is difficult but if you fail to state why your paper is needed and fail to give a specific aim the reviewers and the editor will probably already have made a definite but not decisive decision towards rejection. And be cautious not to mix methods and result section.

Material and methods section serves two purposes. You must describe exactly how the study was conducted in order for the community to evaluate the work and give others the opportunity to repeat your opus. This part should cause little difficulty or discomfort to write, as long as you balance the length of details, not to short and not to lengthy. As a minimum state the material and subjects, procedures, techniques, criteria etc. Remember to point out gold standards and describe in detail the data collection and statistics. Remember that methodological errors could lead to rejection.

It is not possible to recommend the length of the Material and Methods and the Results sections.

Your ambition for the Result section is to present the relevant key data without any explanation, which is left for the discussion part. The results must be presented in sequence (orderly), exactly in the same steps as the method section. Tentative tables and figures should be 
discussed before the result section is formulated, which helps the authors to decide and define which results and which questions are relevant for the manuscript. This is mostly a very enjoyable part of the work with the paper. It is also important to consider the number of tables and figures. If data from your table can just as well be given in a few lines in the manuscript, this is preferred. Do not repeat data from the tables/figures in the text. Tables, make sure the numbers add up. Remember self-explanatory legends and avoid too many abbreviations.

If you submit to an imaging journal you must have good image quality. Low resolution images are not acceptable. In case reports usually the images should be outstanding and clearly demonstrate the topic. Legends must explain the findings in the image. Images not necessary for the manuscript should be omitted.

Discussion needs a special structure. Explanations of the work as well as view and opinions related to the findings in the manuscript are stated in this section. The main purpose is to answer the research aim and question stated in the introduction. Therefore the first paragraph of the discussion should state the main central findings. To make your message clear, the length of the discussion should not be more around 800 words. Limit the discussion to five or six paragraphs without reiteration of results, just adding comments that will be good. First paragraph should state your main result. Next paragraphs give your interpretation of the results and how your findings are in relation to the context of the literature. Address the clinical implications and limitation, preferably each in a paragraph and end with the conclusion (summary) and future directions.

The abstract is a condensed form of the manuscript and should be written as the final part. An effective and sharp abstract improves immensely the changes for a successful manuscript. Do not neglect the abstract; it is the first thing the editor and reviewers read!

\section{The difficult part to accept - is your paper justified?}

Lack of clinical relevance will almost certainly lead to rejection. Also if it adds no new knowledge to the subject you should prepare yourself for rejection. It is often hard for the authors to realize this.

Weak hypothesis and weak aims not related to the results or the conclusion generally also leads to rejection [6].

Should you be given the opportunity to revise your manuscript remember that final acceptance depends on revision according to the instructions and that you address all the issues raised by reviewers and editor.

\section{The part you cannot control}

$\nabla$

Especially if you are writing about a popular topic the editor may already have accepted a similar paper, if that happens yours will probably be rejected.

\section{Is this the right journal?

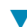

Careful by consider which journal you want to submit to. By ranking the journal by impact factor and starting from the top you may waste valuable time. Sub specialized journals may have lower impact factors but their readers and editors may appreciate your paper more than a journal covering all subspecialties.

\section{The language - fluent English} $\nabla$

Also, usually the paper is written by several authors who are responsible for different sections. Try to have a consistent tune in language, which reflects the main author's personality. If there are major language problems the manuscript will be rejected. We recommend all who are not native English-speaking to consult a fluent English-speaking colleague or a professional scientific writing service. The latter can be done over the internet and usually charge a fee per word. Some offer a free trial e.g., 300 word so you can understand how well they can help you. This is money well spent.

\section{Conclusion $\nabla$}

The conclusion should be written after the abstract and in our opinion this is the very most difficult part to write. Usually, after this exhausting writing process, the authors come to the conclusion that there is nothing more to be said or written. However, it is the abstract and the conclusion that most scientists read of a paper. So take extra time to conclude and summarize the paper and state your final words clear and consistent.

\section{Good Luck!}

\section{Prof. Michael Bachmann Nielsen} mbn@dadlnet.dk

Department of Radiology, Centre of Diagnostic Investigation, Rigshospitalet, University of Copenhagen,

Blegdamsvej 9, DK 2100 Copenhagen, Denmark

\section{References}

1 Calcagno V, Demoinet E, Gollner K, Guidi L, Ruths D, de Mazancourt C. Flows of research manuscripts among scientific journals reveal hidden submission patterns. Science 2012; 338: 1065-9.

2 Khosla A, McDonald RJ, Bornmann L, Kallmes DF. Getting to Yes: The Fate of Neuroradiology Manuscripts Rejected by Radiology over a 2-year Period. Radiology 2011; 260: 3-5

3 http://circ.ahajournals.org/site/misc/stats. xhtml (May 2013)

4 Bachmann Nielsen M, Jaeger K. How to write and publish a successful scientific article. Ultraschall in Med 2007; 28: 472-474

5 Tompson A. How to write an English medical manuscript that will be published and have impact. Surg Today 2006; 36: 407-409.

6 Ehara S, Takahashi K. Reasons for rejection of manuscripts submitted to AJR by international authors. AJR 2007; 188: W113-W116 